\title{
Zuchtversuche mit Heringslarven (Clupea harengus L.).
}

\author{
Von ADOLF KotThaUs. \\ (Aus der Biologisehen Anstalt auf Helgoland.) \\ (Mit 4 Abbildungen im Text.)
}

\section{Inhaltsiibersicht.}

\begin{abstract}
A. Ein leitung
B. Zuchtversuche im Frïhjahr 1936 . . . . . . . . . . . . . . . . . . . . . . . . . 351

I. Vorbereitungen und künstliche Befruchtung . . . . . . . . . . . . . . . . . . . . 351

II. Die vier Hauptversuche . . . . . . . . . . . . . . . . . . . . . . . . . . . . 352

III. Die speziellen Fütterungsversuche . . . . . . . . . . . . . . . . . . . . . . . . 354

C. Zählung von Myomereu und Chordazellen . . . . . . . . . . . . . . . . . . 355

D. Zusammenfassung der Ergebnisse. . . . . . . . . . . . . . . . . . . . . 357
\end{abstract}

\section{A. Einleitung.}

Seit Beginn der Untersuchungen über die Biologie des Herings beanspruchten die "Heringsrassen in erster Linie das Interesse der Forscher. Heincke (2), dem es zuerst gelungen war, die einzelnen „Rassen" auf Grund morphologischer Merkmale einwandfrei zu kennzeichnen, war der Ansicht, daß die „Rassencharaktere" (bestimmte Körperproportionen, Wirbel-, Kielschuppen- und Flossenstrahlenzahl) yon der Umwelt geprägt und mit der Zeit erbfest geworden seien, d. h. daß es sich bei den Heringsrassen um Rassen im heutigen erbbiologischen Sinne handele. In den wesentlichsten Zügen finden wir diese Ansicht auch von SchnakENBECK (13 u. 14) in seinen Arbeiten „Rassenuntersuchungen am Hering" und "Zum Rassenproblem bei den Fischen“ vertreten.

Im Laufe der Zeit wurden aber verschiedentlich Beobachtungen gemacht, die sich mit der HeINCKEschen Ansicht nicht vereinbaren lieben und die darauf hinzudeuten schienen, daß die als Rassenmerkmale angesehenen Eigenschaften nicht erbfest, sondern auch heute noch dem Einflusse äußerer-namentlich thermischer-Faktoren unterlegen sind. Vor allem haben die Untersuchungen RunNsTRøms (12) am norwegischen Frühjahrshering eine besondere Beachtung in den Fachkreisen gefunden; sprechen doch ihre Ergebnisse in hohem Maße dafür, daß die Durchschnittswirbelzahl - das heute gebräuchlichste Charakteristikum der Rassen - von der Temperatur zur Laichzeit abhängig ist.

Inwieweit nun diese gegensätzlichen Anschauungen, die beide ihre Verfechter haben, mit den tatsächlichen Verhältnissen vereinbar sind, wird wohl sehr schwer - vielleicht auch nie - durch Beobachtungen in der freien Natur allein zu entscheiden sein. Eine eindeutige Entscheidung kann hier nur durch das Experiment herbeigeführt werden.

In welcher Weise solche Experimente durchgeführt werden müssen, ergibt sich aus der Fragestellung. Es sind zwei Versuchsreihen notwendig, und zwar müBte bei der ersten künstlich befruchteter Laich einer einzigen „Rasse“ unter verschiedenen, namentlich thermischen, Bedingungen und bei der zweiten Reihe Laich verschiedener Rassen unter gleichen äuBeren Bedingungen zur Entwicklung gebracht werden. Ist die Durchschnittswirbelzahl, die in erster Linie interessiert, erblich fixiert, also von äuberen 
Faktoren unabhängig, so müßte bei der ersten Reihe in allen Versuchen die Durchschnittswirbelzahl die gleiche sein, während das bei der zweiten nicht der Fall sein dürfte. Ist aber die Wirbelzahl durch die äußeren Faktoren zur Entwicklungszeit beeinflußbar, so würden bei den einzelnen Versuchen der ersten Reihe Unterschiede in der Durchschnittswirbelzahl zu erwarten sein, nicht aber bei der zweiten.

Der Durchführung derartiger Experimente stand bisher die Schwierigkeit entgegen, daß es nicht gelingen wollte, den Hering künstlich vom Ei bis zu einem Stadium zu züchten, auf welchem die "Rassencharaktere" bereits definitiv festgelegt sind, d. h. bis zu einer Länge von etwa $30-35 \mathrm{~mm}$. Es gelang nicht, die Larven länger als bis zur Aufzehrung des Dottersackes zu halten; auf diesem „kritischen“ Stadium gingen die Larven regelmäßig ein.

Diese Schwierigkeit mußte also zunächst überwunden werden, ehe an die Durchführung der eigentlichen Experimente gedacht werden konnte. Diesem Ziele dienten in der Hauptsache die seit einigen Jahren an der Biologischen Anstalt auf Helgoland unter Leitung von Professor Dr. Mielcis und nach dessem Tode von Professor Dr. HAGMeier durchgeführten Heringszuchtversuche, mit welchen sich nacheinander die wissenschaftlichen Mitarbeiter W. ERdManN, W. MarX und der Verfasser befaßt haben. Ueber die Ergebnisse meiner eigenen Versuche soll im folgenden berichtet werden. Dabei kann ich schon auf die Fortsetzung der Versuche hinweisen, wobei es meinem Nachfolger Dr. ScHACH glückte, eine größere Anzahl von Heringslarven über das kritische Stadium hinaus zu züchten. In der folgenden Arbeit dieser Zeitschrift wird Dr. ScHACH selbst über seine Versuche berichten. Hier scheint es angebracht, kurz auf die früheren Versuche einzugehen.

Bereits 1876 wurden von H. A. MeYer (7) in Kiel die ersten Versuche einer künstlichen Aufzucht des Herings angestellt. MEYer benutzte für seine Versuche künstlich besamten Heringslaich aus der Schlei und von Korsör. Bei der künstlichen Befruchtung wurden sowohl die Männchen wie auch die Weibchen unter Wasser abgestreift; etwa 15 Minuten nach dem Abstreifen wurde das spermahaltige Wasser durch frisches ersetzt. Die Erbrütung des Laiches erfolgte in einem mit Gaze bespannten Kasten, der in der Schlei etwa $50 \mathrm{~cm}$ unter der Wasseroberfläche versenkt gehalten wurde. Diesem Kasten wurden zu verschiedenen Zeiten Proben zur Kontrolle der Entwicklungsvorgänge im Ei, die von C. KUPFFrR ( 5 u. 6) angestellt wurden, und für einzelne Versuche, bei denen es galt, den Einfluf thermischer Faktoren auf die Entwicklungsdauer festzustellen, entnommen. Keine der von MEYER erhaltenen Larven überstand den "kritischen" Punkt.

ERdMans benutzte 1934 zur Zucht natïrlich abgelegten Laich aus dem nordfriesischen Wattenmeer, welcher mittels Flugbootes nach Helgoland gebracht wurde. Zum größeren Teil waren die Eier auf Fucus, zum geringeren Teil auf Zostera abgelegt. Die Erbrütung des Laiches fand teils im Austernzucht-Aquarium, teils im Keller des Hafenlaboratoriums statt. Zwei der im Keller geschlüpften Larven konnten bis zu einer Länge von etwa $15 \mathrm{~mm}$ gehalten werden, alle übrigen Larven gingen auf dem kritischen Punkte ein. Nach ERDMANNS Angaben sollen Monadinen zur Fütterung der Larven ungeeignet sein, weil diese Algen nach seiner Ansicht im Darme weiterleben und assimilieren sollen. Häufig im Darm der Larven angetroffene Gasblasen, die in der Hauptsache für das Eingehen der Tierchen verantwortlich zu machen sind, führt ERDMANN einerseits auf die Assimilationstätigkeit der Monadinen im Darm der Larven zurück, andererseits aber auch darauf, daß nach seinen Beobachtungen die Larven kleine, im Wasser verteilte Luftbläschen verschlucken sollen. Die Ansicht Erdmanns, die Larven seien gegen Temperaturschwankungen sehr empfindlich, widerspricht dem Befunden MEYers, und anch ich konnte bei meinen Versuchen nur das Gegenteil bestätigt finden.

MARX ${ }^{1}$ ), der im Jahre 1935 die Zuchtversuche weiterführte, benutzte zur Zucht künstlich befruchtete Heringseier des frühjahrslaichenden $\mathrm{Em} \mathrm{shering} \mathrm{s,} \mathrm{die} \mathrm{bei} \mathrm{der}$ ersten Versuchsreihe auf steriler Glas wolle, bei der zweiten auf Glasplatten abgelegt wurden. Letzteres Verfahren erwies sich nicht als giinstig, da nur ein verschwindend kleiner Prozentsatz der Eier ausschlüpfte. Die Erbrütung des Laiches fand in Behältern statt, welche an verschiedenen Stellen des Hafenlaboratoriums (Austernzucht-Aquarium, Sortierraum, Kurssaal, Keller und im Freien) aufgestellt waren. In keinem der Behälter konnten während der Erbrütung konstante Temperaturen erzielt werden. Fütterungsversuche wurden mit Musehel- und Schneckenlarven, Nauplien von Copepoden und mit gemischtem Plankton durchgeführt. Bei beiden Versuchen gelang es, eine Anzahl der Larven - etwa 20-30 Stück - über das kritische Stadiurn hinaus zu halten, deren maximale Größe bei einem Alter von 5 Wochen auf etwa $30 \mathrm{~mm}$ geschätzt wurde. Als Nahrung

1) Arbeitsanweisung für die künstliche Heringszncht (Unveröffentlichter Bericht, 1936). 
konnten im Darm nachgewiesen werden: 1. Larven von Nacktschnecken und 2. Nauplien von Copepoden. Für eine erfolgreiche Zucht sind nach MARX Auffassung drei Punkte zu beachten. Es ist 1. erforderlich, daß die Zuchtbecken undurchsichtige Wände haben, 2. daß die Zuchtbecken mit fließendem Wasser versehen sind und 3. daß genügend geeignete Nahrung zur Verfügung steht.

Mit diesen Erfahrungen meiner Vorgänger ging ich nun im Frühjahr 1936 selbst an die Aufgabe heran. Die wesentlichsten Ergebnisse werden auf den folgenden Seiten geschildert.

\section{B. Die Zuchtversuche im Frihjahr 1936.}

\section{Vorbereitungen und künstliche Befruchtung.}

Zur Erbrütung des Laiches und zur Hälterung der geschlüpften Larven wurden in der Hauptsache etwa 165 Liter fassende, aus säurefestem Material bestehende, zylindrische Gefäße (sog. Tonrohre) benutzt. Diese Gefäße wurden teils mit filtriertem, teils mit unfiltriertem Seewasser gespeist. Bei einigen Rohren (Hauptversuch I, III und IV) wurde das frische Wasser etwa $20 \mathrm{~cm}$ über dem Boden der GefäBe eingeleitet, während es bei anderen (Hauptversuch II) einfach von oben längs der Wand einfloß. Sämtliche Abflüsse waren durch kleine, mit Gaze bespannte Filterbecher gesichert. Der Zufluß des Wassers war so eingestellt, daß sich der Inhalt der Tonrohre inverhalb 24 Stunden etwa 3- bis 4-mal erneuerte. Einen stärkeren Durchstrom, der mitunter zur Temperaturregulierung sehr erwünscht gewesen wäre, konnten die Filter nicht bewältigen. Die zum Hauptversuch I benutzten Rohre konnten außerdem noch durchlüftet werden. Wegen der großen Zahl der geschlüpften Larven war es notwendig, einen Teil derselben in andere Gefäße, teils Tonrohre, teils große Aquarienbecken, überzuführen und darin weiter zu zuichten. Auch diese Gefäße waren mit fließendem Wasser und durch Filter gesicherte Abläufe versehen.

Es wurden 4 Hauptversuche unter verschiedenen Temperatur- und Belichtungsbedingungen durchgeführt, und zwar wurden diese Unterschiede dadurch erzielt, daf die zur Zucht benutzten Gefäße an verschiedenen Stellen des Hafenlaboratoriums aufgestellt wurden. Zum Versuch I wurden zwei Tonrohre im Austernzucht-Aquarium so aufgestellt, daß sie fast während der ganzen Tagesstunden vom Sonnenlicht getroffen wurden. Die zum Versuch II benutzten Rohre, die gleichfalls im Austernzucht-Aquarium Aufstellung fanden, wurden nur während der späten Morgen- und frühen Nachmittagsstunden direkt von der Sonne beschienen. Bei Versuch III standen die dazu benutzten GefäBe im Sortierraum des Hafenlaboratoriums in einiger Entfernung vom Fenster, so dab direktes Sonnenlicht niemals in sie hineinfallen konnte; zudem war das Fenster noch durch Zuggardinen abblendbar. Der Versuch IV wurde bei tagsüber nur sehr schwachem Licht im Keller durchgeführt. Bei keinem der vier Versuche war es möglich, konstante Temperaturen in den Zuchtgefäßen zu halten, vor allem stiegen die Temperaturen in den im Austernhaus stehenden Rohren tagsüber ganz beträchtlich an.

Zur Fütterung der Larven in den Hauptversuchen warde gemischtes, auf der Reede von Helgoland gefischtes Plankton, das zuvor durch Gaze 3 gesiebt wurde, um die größeren Planktonten zurückzuhalten, benutzt. Besonders wurde darauf geachtet, daß die Temperatur des planktonhaltigen Wassers vor der Ueberführung in die Zuchtgefäße derjenigen in letzteren gleich war. Die Ueberleitung des Nährwassers geschah mittels Hebers, um eine Schädigung sowohl der Planktonten als auch der Heringslarven zu vermeiden.

Um besondere Fütterungsversuche durchführen zu können, war es erforderlich, durch Kulturen die nötige Menge an Nährorganismen bereitzustellen. Larven von verschiedenen Aeolis- und Doris-Arten konnten ohne Schwierigkeit aus im Freien oder im Aquarium abgelegten Laich gezüchtet werden, die sich bei Fütterung mit Monadinen bequem halten ließen. Mytilus-Larven konnten jederzeit durch künstliche Befruchtung nach der von HAGMEIER (1) angegebenen Methode in genügender Zahl gewonnen werden. Nauplien von Copepoden in genügender Menge in Reinkultur zu züchten, erschien in Anbetracht der geringen Nachkommensehaft eines einzelnen Copepoden als ziemlich aussichtslos. Es ließ sich jedoch erreichen, Nauplien in großer Zahl mit geringer Beimischung anderer kleiner Planktonten auf folgende Art und Weise zu erhalten. Auf der Reede gefischtes grobes Plankton wurde durch Gaze 3 gesiebt und die auf der Gaze zurückbleibenden Copepoden wurden in ein Tonrohr gegeben(an Copepoden im Plankton hauptsächlich: Temora, Pseudocalanus und Acartia). Schon nach 1-2 Tagen waren in dieser Kultur große Mengen von Nauplien vorhanden, die dann durch einen Heber mit vorgeschaltetem Gazefilter 
(Gaze 14) abgesaugt werden konnten, während die Copepoden selbst zurückgehalten wurden. Monadinenkulturen wurden in der üblichen Weise angesetzt.

Für die Fütterungsversuche wurden kleine, etwa 3 bis 5 Liter fassende Vollglasaquarien oder Brutgläser benutzt, in denen das Wasser durchlüftet werden konnte und alle 3 Tage durch sorgfältig filtriertes frisches Wasser ersetzt wurde.

Wie im Jahre 1935, so wurde auch im Jahre 1936 zur Zucht wieder künstlich besamter Laich des oberhalb Emden und im Dollart in Stellnetzen gefangenen frühjahrslaichenden Emsherings benutzt. Die künstliche Befruchtung in Emden am 9. April wurde in der Weise durchgeführt, daß zunächst ein Weibchen über Glaswolle (sog. Engelshaar), welche in einem etwa $\mathrm{zu} 3 / 4_{4}$ mit reinem Nordseewasser gefülltem Brutglase ausgebreitet war, abgestreift und darauf die Milch eines Heringsmännchens darüber gespritzt wurde. Durch leichtes Schwenken mit dem Glase wurde eine gleichmäßige Verteilung des Spermas im Wasser erzielt. Etwa 20 bis 30 Minuten nach diesem Vorgange und nachdem angenommen werden konnte, dab die Befruchtung vollzogen sei, wurde das spermahaltige Wasser durch frisches, ebenfalls reines Nordseewasser, ersetzt. Während der 14-stündigen Rückfahrt nach Helgoland wurde das Wasser noch dreimal gewechselt, außerdem wurde schon bei der Heimfahrt versucht, Temperaturunterschiede in den einzelnen Brutgläsern zu erzielen, was auch tagsüber durch Aufstellung der Gläser an verschiedenen Stellen des Schiffes (frei an Deck, im Sonnen- und Windschatten und in Aquarientanks mit fließendem Wasser) einigermaßen gelang, dagegen sanken die Temperaturen während der Abend- und Nachtstunden bis zur Ankunft auf Helgoland um Mitternacht in allen Gefäßen auf etwa 5.2 bis $5.4^{\circ} \mathrm{C}$.

Sogleich nach der Ankunft auf Helgoland wurde der Laich in die zu dem späteren Versuch III benutzten Rohre gegeben, da hier die Temperaturen denen in den Brutgläsern am nächsten (um $2^{\circ}$ höher) lagen. Im Laufe der folgenden Tage wurde dann der Laich gleichmäßig auf die übrigen Zuchtgefäße verteilt, und zwar so, daß die Glaswolle mit dem Laich zunächst in kleinere Gläser gegeben und dann in die BestimmungsgefäBe eingehängt wurde, bis die Temperaturen in beiden Gefäßen ausgeglichen waren.

\section{Die vier Hauptversuche.}

a) Versuch I.

Die erste Beobachtung über die Entwicklung des Embryos im Ei wurden am 10. April - 36 Stunden nach der Befruchtung - gemacht. Es zeigte sich dabei zunächst, daß eine Anzahl der Eier abgestorben oder zum Teil nicht befruchtet war. Das scheint mir einerseits darauf zurückzuführen zu sein, daf namentlich die auf den äußeren Strähnen der Glaswolle sitzenden Eier auf dem Transport von Emden nach Helgoland bei sehr bewegter See durch die ständige Reibung an der Wand des Gefäßes und den daran auch noch klebenden Eiern gelitten haben, andererseits war die Befruchtung bei einer Anzahl von Eiern wohl deshalb unterblieben, weil die Eier an manchen Stellen dicht übereinander lagerten, so daß der Zutritt der Spermien zu den zu unterst liegenden Eiern verwehrt war. Im Vergleich zur Gesamtmenge war jedoch die Zahl der abgestorbenen Eier ziemlich unbedeutend.

Bei den lebenden Eiern war bei der ersten Beobachtung die Keimscheibe als dicke Kappe über dem Dotter zu erkennen; am folgenden (2. Tag nach der Befruchtung) Tage umspannte der Embryo schon etwa zur Hälfte den Dotter. Am 3. Tage berührten sich bereits der Kopf und die Schwanzspitze, zum Teil waren sie schon aneinander vorbeigewachsen. Bereits am 4. Tage konnten die ersten Bewegungen und der Herzschlag beobachtet werden. Am 5. Tage begann die Pigmentierung der Augen, die am 6. Tage bei allen Embryonen eingetreten war. Die Bewegungen der Larven im Ei wurden immer lebhafter und waren am Laich, der sich am 7. Tage gelblich verfärbte, mit bloßem Auge wahrzunehmen.

Die ersten Larven wurden am 17. April, also am 8. Tage nach der Befruchtung, frei. Die Mehrzahl schlüpfte aber am 9. und der Rest am 10. Tage. Im Folgenden wird bei der Altersangabe der 18. April (9. Tag nach der Befruchtung) als Geburtsdatum zugrunde gelegt. Insgesamt schlüpften etwa $3-5000$ Larven. Die Temperatur während der Inkubationszeit schwankte zwischen $8.5^{\circ}$ und $16.8^{\circ}$; im Mittel betrug sie $12.3^{\circ}$. (vergleiche Abb. 1).

Bereits am 2. Tage nach dem Schlüpfen war bei den meisten Larven äußerlich nichts mehr vom Dottersack zu erkennen, während Reste des Dotters noch bis zum 9. Tage am und im Darm nachweisbar waren. Die Mundöffnung brach am 2. Tage durch. 
Am 3. Tage traten zum ersten Male Larven mit einer kleinen Gasblase im Enddarm auf und verursachten, daß die Larven hilflos an der Oberfläche schwammen und bald eingingen. Das Auftreten von Larven mit derartigen Gasblasen im Darm wurde von Tag zu Tag häufiger und erreichte am 29. und 30. April (11. und 12. Tag) seinen Höhepunkt und damit gleichzeitig seinen Abschluß. Nach diesem großen Sterben wurden keine Larven mehr mit Gasblasen angetroffen. Die Tierchen, die in der Folgezeit eingingen, lagen stets am Boden. Von der großen Zahl der Larven waren Ende April nur noch etwa 200-300 Stück übrig geblieben, von denen in den folgenden Tagen immer noch einige eingingen, bis am 20. Mai (32. Tag) auch die letzte Larve aus diesem Versuch gestorben war.

Vom 10. Tage ab konnte bis zum Schluf des Versuches bei allen Larven Nahrung im Darm festgestellt werden; allerdings nur Nauplien von Copepoden und eine grüne strukturlose Masse, wie sie auch von LEBour (8-11) und P. JESPERSEN (4) immer wieder im Darm der jüngsten Larven gefunden wurde. LEBour vermutet, daß es sich bei diesen "green food remains" um Reste pflanzlichen Nannoplanktons handele. Mit Sicherheit kann aber über das Wesen dieser grünen Masse nicht entschieden werden.

Bei der Untersuchung der Larven mit Gasblasen im Darm fand ich in allen Fällen den Darm vollkommen leer; die Assimilation von Phytoplanktonten kann also nicht für das Auftreten der Gasblasen verantwortlich gemacht werden. Trotz häufiger und längerer Beobachtungen konnte ich auch niemals feststellen, daß die Larven nach Luftbläschen schnappten. Schließlich habe ich mich auch davon überzeugt, daß die Larven keineswegs unter Sauerstoffmangel litten; denn in den durchlüfteten Gefäßen war das Wasser mit Sauerstoff übersättigt, während in den übrigen Gefäßen der Sauerstoffgehalt des Wassers normal war. Auffällig war es, daf das Auftreten der Gasblasen im Darm zu bestimmten Tageszeiten (in den späten Nachmittagsstunden zwischen 17 und $19 \mathrm{Uhr}$ ) besonders häufig war, während $\mathrm{zu}$ anderen Tageszeiten solche Fälle nur vereinzelt festzustellen waren, und daß von einem bestimmten Zeitpunkte $a b$, der in der Regel mit dem Verschwinden der letzten Reste des Dotters zusammenfiel, keine Gasblasen mehr auftraten. Man könnte vielleicht auch vermuten, daß es sich bei diesen Gasblasen um Gase handelt, die bei dem Abbau des Dottermaterials entstehen. Genaueres läßt sich aber heute noch nicht sagen. Erst wenn man genau weiB, um was es bei diesen Blasen handelt - vielleicht wird sich das durch Mikrogasanalyse feststellen lassen, - wird man der Haupttodesursache der Larven wirksam begegnen können.

\section{b) Versuch II.}

Die erste Entwicklung verlief ähnlich der in Versuch I. Der Prozentsatz toter Eier war hier noch geringer als dort. Am 3. Tage umspannten die Embryonen den Dotter etwa zur Hälfte. Am 5. Tage begannen sich die Embryonen zu bewegen. Am 7. Tage waren die Augen bei fast allen Larven pigmentiert, und die Bewegung der Larven wurden immer lebhafter. Am 9. Tage trat die Verfärbung des Laiches, das Zeichen für das kurz bevorstehende Schlüpfen, ein. In den späten Abendstunden wurde hier die erste Larve beobachtet; am 10. Tage waren etwa 30 Larven geschlüpft, während die große Masse am 11. und 12. Tage frei wurde (der 11. Tag wird als Geburtsdatum angesehen). Während der Entwicklung im Ei schwankten die Teimperaturen zwischen $8^{\circ}$ und $14.3^{\circ}$ und betrugen im Mittel etwa $10.7^{\circ}$ (Abb. 1). Insgesamt wurden aus dieser Zucht etwa 3-4000 Larven erhalten. Aeußerlich war der Dottersack bei fast allen Larven am 4 . Tage verschwunden, während Reste desselben noch bis zum 14. Tage nachweisbar waren.

Am 5. Tage traten in diesem Versuche die ersten Larven mit Gasblasen im Darm auf. Hauptsächlich aber trat das große Sterben am 25 . und 26. April (14. und 15. Tag), also zeitlich etwas später als beim vorigen Versuch, dafür aber sehr viel stärker, ein. Am 15. Tag lebten noch etwa $30-50$ Larven, von denen in den folgenden Tagen aber noch einige eingingen. Die letzten Larven, die

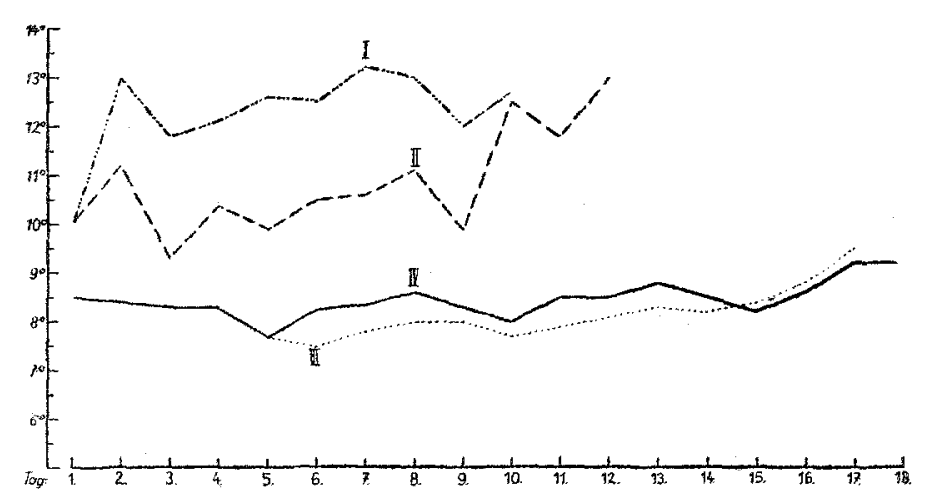

Abb. 1. Durchschnitts-Temperaturen (in Cels.) in den Hauptzuchtversuchen I-IV bis zum Schlïpfen der Larven. 
eine maximale Größe von etwa $25 \mathrm{~mm}$ erreicht hatten, wurden am 13. Juni, also am 54. Tage nach dem Schlüpfen, fixiert. Ich zweifle nicht daran, daß sich diese restlichen Larven bei $\mathrm{g} u ̈ \mathrm{nstig}$ eren Bedingungen ohne Schwierigkeiten noch hätten weiter halten lassen; allein die Temperaturen im Austern-Aquarium wurden wegen der beginnenden Austernzucht so hoch, daß es doch nur kurze Zeit gedauert hätte, bis diese Larven der Hitze erlegen wären.

Die erste Nahrungsaufnahme konnte am 15. Tage nach dem Schlüpfen beobachtet werden, und zwar anfänglich auch wieder nur Nauplien und besagte grüne Masse. In der letzten Zeit wurden aber keine Nauplien mehr aufgenommen, obgleich diese in großen Mengen vorhanden waren, sondern der Darm der Larven war gefüllt mit Schwärmern von Grünalgen (wahrscheinlich von Enteromorpha, die sich an den Wänden der Zuchtgefäße in großen Mengen angesiedelt hatten).

\section{c) Versuch III.}

Die Entwicklung ging hier unter dem Einfluf geringerer Temperaturen viel langsamer vonstatten als in den beiden vorher besprochenen Versuchen. Erst am 6. Tage waren die ersten Bewegungen der Embryonen zu erkennen. Das Augenpigment trat erst am 10. Tage auf, und am 14. Tage wurde die erste Larve gesehen. In der Hauptsache aber schlüpften die Larven am 17. Tage (Geburtsdatum). Auch aus dieser Zucht wurden einige Tausend Larven erhalten. Während der Entwicklung im Ei unterlagen die Temperaturen nur geringen Schwankungen $\left(7.2^{\circ}\right.$ bis $\left.9.6^{\circ}\right)$, im Durchschnitt lagen sie bei $8.7^{\circ}$ (vergl. Abb. 1).

Während der Dottersack äußerlich am 7. Tage nicht mehr vorhanden war, ließen sich Reste des Dotters bis zum Schluß des Versuches am und im Darm nachweisen.

Am 8. und 9. Tage traten die ersten Larven mit Gasblasen auf. Bis zum 14. Tage war die Zahl der eingegangenen Larven verhältnismäßig gering. In der Nacht vom 14. zum 15. Tage gingen in allen drei Tonrohren bis auf etwa 15 Stück alle Larven ein, und dieser Rest überlebte auch nicht mehr den Abend des 15. Tages. Für dieses Sterben konnte aber nicht das Vorhandensein von Gasblasen im Darm verantwortlich gemacht werden, da die Larven alle auf dem Boden lagen. Die Ursache für das Eingehen konnte nicht mehr festgestellt werden.

Nahrungsaufnahme konnte bei den Larven dieser Versuchsreihe in keinem Falle nachgewiesen werden.

$$
\text { d) Versuch IV. }
$$

Da der Laich bis zum 6. Tage mit in den Gefäßen des Versuches III untergebracht war, so verlief die Entwicklung bis dahin ähnlich wie im vorigen Versuch. Erst nach der Ueberführung des Laiches in die definitiven Gefäße trat eine geringe Verzögerung der Entwicklung ein. Eine leichte Verfärbung des Laiches konnte schon am 13. Tage festgestellt werden, während die erste Larve erst am 16. Tage beobachtet wurde. Am 17., in der Hauptsache aber am 18. Tage schlüpfte die Mehrzahl der Larven (18. Tag - Geburtsdatum). Die Temperaturen schwankten bis dahin zwischen $7.5^{\circ}$ und $9.6^{\circ}$ und betrugen im Mittel 8.3\%. (Abb. 1.)

Am 6. Tage nach dem Schlüpfen war im allgemeinen vom Dottersack nichts mehr zu sehen, aber auch hier, wie bei Versuch III, waren Dotterreste bis zum Schluß des Versuches nachweisbar. Am 6. Tage wurde auch bereits die erste Larve mit einer Gasblase im Darm beobachtet. Das Sterben war allerdings in den nächsten Tagen noch recht gering. Am 14., 15. und 16. Tage ging jedoch die Mehrzahl der Larven ein und von den etwa 30 zurückgebliebenen Tieren war am 20. Tage kein einziges mehr am Leben. Nahrungsaufnahme konnte auch bei den Larven dieses Versuches nicht festgellt werden.

\section{Die speziellen Fütterungsversuche.}

Die Fütterungsversuche wurden alle in der folgenden Weise durchgeführt: etwa 50 bis 100 Larven wurden kurz nach dem Schlüpfen den Hauptzuchtgefäßen entnommen und in Brutgläser oder kleine Aquarien gesetzt. Das Wasser wurde in diesen Gefäßen durchlüftet und alle drei Tage mittels Hebers mit vorgeschaltetem Filter abgesaugt und durch filtriertes ersetzt. Den Gläsern wurde sogleich eine ausreichende Menge von Nährorganismen aus den Kulturen zugesetzt. Jeden Tag wurden etwa 5-10 Larven aus jedem 
Glase zur Kontrolle der Nahrungsaufnahme herausgefangen. Insgesamt wurden 7 Fütterungsversuche. mit Larven von Nacktschnecken, 3 mit Larven von Mytilus, 3 mit Monadinen und einige mit Nauplien angestellt. Austernlarven konnten erst gegen Ende Mai erhalten werden, als die Mehrzahl der Larven bereits eingegangen war; die mit Austernlarven beabsichtigten Fütterungsversuche mußten daher ausfallen.

Im einzelnen brauche ich nicht auf die Versuche einzugehen, da sie alle das gleiche negative Ergebnis zeitigten. Mit Ausnahme einer einzigen Larve, bei welcher die bekannte grüne Masse im Darm festgestellt werden konnte, war in keinem Falle eine Aufnahme der zur Verfügung stehenden Nährorganismen nachzuweisen, obgleich letztere bis zum Ende eines jeden Versuches in genügender Menge vorhanden waren. Die Larven gingen auch hier ein, sobald die letzten Reste des Dotters aufgezehrt waren.

In diesen Gläsern konnte ich bei der Untersuchung einer Anzahl toter Larven und solcher, die kurz vor dem Verenden standen, feststellen, daß diese von einer Unzahl einer hypotrichen Ciliatenart (vermutlich zur Familie der Stylonichidae gehörig) befallen waren. Diese Ciliaten saßen nicht nur außen am Körper, sondern auch in der Mundhöhle, im Darm und in der Chorda. Zum Teil waren die toten Larven bis auf das Skelett "abgenagt". Bei einer noch frei schwimmenden Larve fand ich ebenfalls einen Befall dieser Ciliaten. Man könnte sich vorstellen, daß während der Nacht, wo sich die Larven über das ganze Gefäß verteilen - bei Tage halten sie sich gewöhnlich an der Oberfläche auf und dabei auch zum Boden der Gefäße kommen, sich die Ciliaten auf die Larven, vor allem auf die schon etwas schwachen, "stürzen“" und bei lebendigem Leibe „auffressen“.

Obgleich im Bodensatz aller, auch zu den Hauptzuchtversuchen benutzter Behälter, diese Ciliaten vorgefunden wurden, so habe ich jedoch keine Anhaltspunkte dafür, daß3 sie in bedeutendem Maße für das Sterben der Larven verantwortlich zu machen sind, wenn sie auch - was nicht zu bezweifeln ist - einen geringen Teil dazu beigetragen haben werden.

\section{Zählung von Myomeren und Chordazellen.}

Von vorneherein konnte es nicht als ausgeschlossen gelten, daß ein bestimmtes abhängiges Verhältnis zwischen der Zahl der Chordazellen, der Myomeren und der Wirbel bestünde. Um diese Frage zu klären, wurden im Anschluß an die tägliche Kontrolle über die Nahrungsanfnahme der Larven - soweit dazu Zeit zur Verfügung stand - an dem gleichen Material Chordazellen- und Myomerenzählungen gemacht. Wegen der lebhaften Bewegungen der Larven war es nur möglich diese Zählungen an betäubten Larven durchzuführen. Als Betäubungsmittel wurde eine $0,5 \%$-ige wässerige Lösung von Chloreton

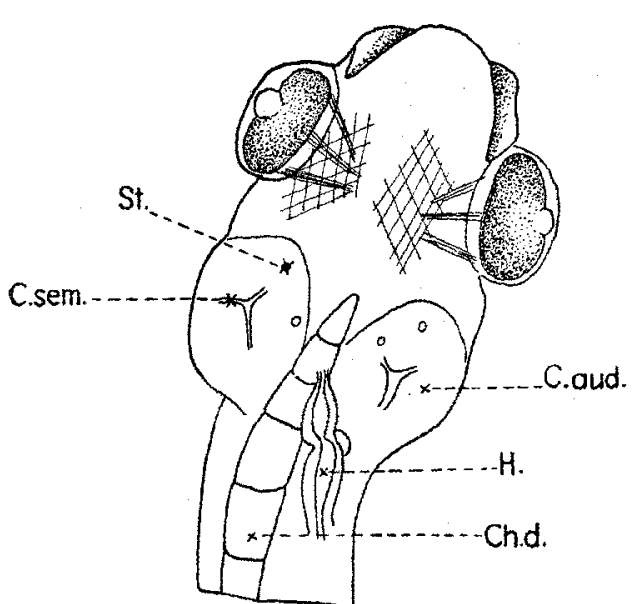

Abb. 2. Chordaspitze einer Heringslarve von $9 \mathrm{~mm}$ Körperlänge; Ventralansicht des Kopfes.

Abkürzungen : C. aud. $=$ Capsula auditiva; Ch. d. $=$ Chorda dorsalis; C. sem. $=$ Canalis semicircularis; H. $=$ Herz; St. $=$ Statolith .

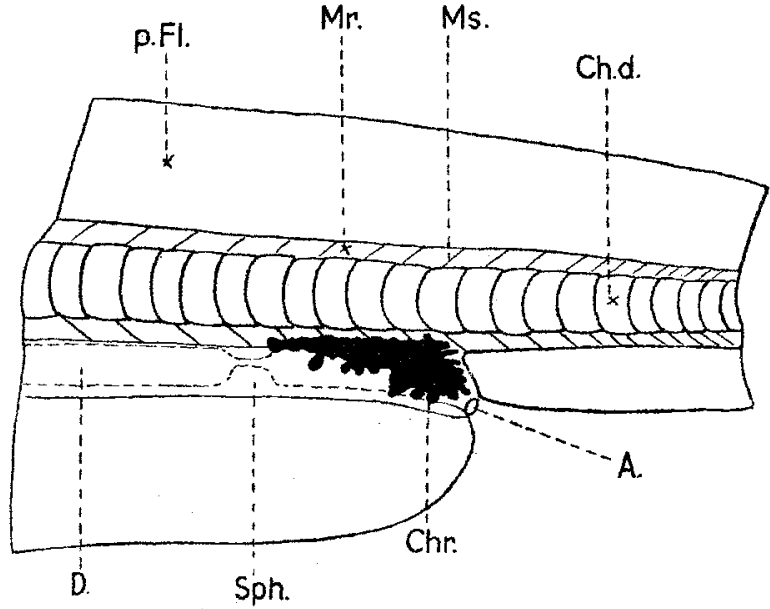

Abb. 3. Normal ausgebildeter Abschnitt der Chorda dorsalis einer Heringslarve von $9 \mathrm{~mm}$ Körperlänge.
Abkürzungen : A. $=$ After; Ch. $d .=$ Chorda dorsalis; Chr. = Chromatophor D. = Enddarm; Mr. = Myomer; Ms. $=$ Myosepte; p. Fl. = primordialer Flossensaum; Sph. = Schließmuskel des Enddarmes. 
(Azetonchloroform) benutzt, von der einige Tropfen dem Seewasser zugesetzt wurden. Bereits tote Larven eigneten sich zur Zählung nicht mehr, da deren Muskulatur undurchsichtig geworden war und die Chordazellen und die Myomeren nicht mehr deutlich erkennen ließ.

Was die Chorda anbetrifft, so zeigt sie das typische Bild der Geldrollenform Abb. 2-4). Allerdings treten häufig, namentlich im hinteren Chordaabschnitt (Abb. 4) unregelmäßig gestaltete Zellen auf; so sehen wir auf unserer Abbildung zwei keilförmig

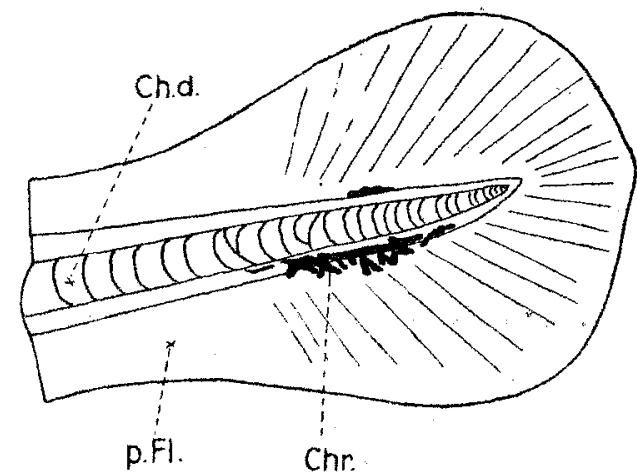

Abb. 4. Schwanzende einer Heringslarve von $9 \mathrm{~mm}$ Körperlänge, unregelmäßig gestaltete Zellen der Chorda dorsalis zeigend. Abkürzungen: $\mathrm{Ch} . \mathrm{d} .=\mathrm{Cborda}$ dorsalis; Chr. = Chromatophor; p. Fl. = primordialer Flossensaum. einschneidende Zellen, in anderen Fällen ist die Vakuolisierung der Chordazêllen nicht so weit fortgeschritten, als daß die Zellen die übliche Größse erlangt hätten. So findet man dann häufig an Stelle einer Zelle zwei oder gar drei, oder es kann schlieBlich der letzte Chordaabschnitt ganz unregelmäßig aufgebaut sein. Für die Zählungen wurden nur solche Larven benutzt, deren Chordazellen alle normal ausgebildet waren. Daraus erklärt sich auch zum Teil die geringe Zahl der Zählungen; denn die Zahl der Larven mit anormal ausgebildeten Chordazellen war keineswegs gering.

Die Zahl der Chordazellen schwankte zwischen 91 und 124; im Mittel betrug sie 111,3. Irgend eine Abhängigkeit der Chordazellenzahl von der Temperatur zur Entwicklungszeit konnte nicht festgestellt werden.

Das Zählen der Myomeren gestaltete sich ziemlich schwierig, da die Myosepten im caudalen Körperabschnitt im allgemeinen recht undeutlich waren. Etwa von der Mitte zwischen After und dem Körperende hörte die Segmentierung auf, wenigstens soweit man das bei Betrachtung der Larven in toto beurteilen kann. Ob tatsächlich der letzte Körperabschnitt unsegmentiert bleibt oder ob die Myosepten in dieser Körperregion nur zu zart sind, um obne weiteres erkannt zu werden, vermag nur an Hand von Längsschnittserien - leider fehlte die Zeit, solche herzustellen - entschieden werden. - Die Myomeren wurden nur bei solchen Larven gezählt, bei welchen zuvor die Zahl der Chordazellen festgestellt worden war.

Wie die nachfolgende Aufstellung zeigt,

$\begin{array}{cc}\begin{array}{c}\text { Zahl } \\ \text { der Myomeren }\end{array} & \begin{array}{c}\text { Zahl } \\ \text { der Chordazellen }\end{array} \\ 63 & 107 \\ 61 & 111 \\ 61 & 119 \\ 60 & 107 \\ 60 & 111 \\ 60 & 115 \\ 60 & 117 \\ 59 & 92 \\ 59 & 93 \\ 59 & 102 \\ 59 & 102 \\ 59 & 105 \\ 59 & 108 \\ 59 & 112 \\ 59 & 112 \\ 59 & 112 \\ 59 & 113 \\ 59 & 114 \\ 59 & 115\end{array}$

$\begin{array}{cc}\begin{array}{c}\text { Zahl } \\ \text { der Myomeren }\end{array} & \begin{array}{c}\text { Zahl } \\ \text { der Chordazellen }\end{array} \\ 59 & 117 \\ 59 & 122 \\ 59 & 123 \\ 58 & 102 \\ 58 & 104 \\ 58 & 106 \\ 58 & 114 \\ 58 & 116 \\ 58 & 118 \\ 58 & 121 \\ 58 & 124 \\ 56 & 96 \\ 56 & 114 \\ 55 & 108 \\ 55 & 112 \\ 55 & 122 \\ (51) & 119 \\ (51) & 113\end{array}$

schwankt die Zahl der Myomeren zwischen 55 und 63 (Durchschnitt: 58,6), wobei zwei Werte von 51 unberücksichtigt bleiben sollen, da sie bestimmt zu niedrig liegen (die 
Myomeren waren bei diesen Larven im letzten Körperabschnitt nicht deutlich zu erkennen). Wie die Tabelle weiter zeigt, steht die Zahl der Myomeren zu derjenigen der Chordazellen in keinem bestimmten Verhältnis. - Die Durchschnitts-Myomerenzahl liegt um etwa 4 höher als die mittlere Wirbelzahl des Emsherings. Wenn man nun bedenkt, daß 2 bis 3 Segmente zur Bildung des Urostyls beitragen und wenn man ferner annimmt, daß eventuell ein Körpersegment dem Schädel angegliedert wird, so würde der Wert für die Restmyomeren in etwa mit der erwarteten Wirbelzahl übereinstimmen. Auf Grund der verhältnismäßig geringen Zahl der Zählungen läßt sich sicheres in dieser Beziehung natürlich nicht sagen. Immerhin würde es von großem Interesse sein, wenn eine derartige $\mathrm{Be}-$ ziehung zwischen Myomeren- und Wirbelzahl nachgewiesen würde, hätte man dann doch ein Mittel, auch bei den jüngsten Larven, deren Wirbel noch nicht ausgebildet sind, durch Zählung der Myomeren die Zahl der Wirbel zu bestimmen.

\section{Zusammenfassung der Ergebnisse.}

Ueber die Beobachtungen während der Heringszuchtversuche sei zusammenfässend folgendes festgestellt:

1. Die Entwicklungsdauer im Ei betrug bei einer Durchschnittstemperatur von

a) $12.3^{\circ} 7$ bis 9 Tage (Versuch 1)

b) $10.7^{\circ} 10$ bis 12 Tage (Versuch II)

c) $8.7^{\circ} 14$ bis 18 Tage (Versuch III)

d) $8.3^{\circ} 17$ bis 20 Tage (Versuch IV)

2. Die Dauer vom Schlüpfen bis zum äußerlichen Verschwinden des Dottersackes einerseits und bis zur völligen Resorption des Dotters andererseits betrug bei einer Durchschnittstemperatur von

a) $12.8^{0} 3$ bzw. 9 Tage (Versuch I)

b) $12.0^{0} 5$ bzw. 14 Tage (Versuch II)

c) $10.7^{\circ} 7$ bzw. 16 Tage (Versuch III)

d) $10.3^{0} 7$ bzw. 20 Tage (Versuch IV)

3. Nur in den Versuchen (I und II), wo die Zuchtgefäße vom Sonnenlicht getroffen werden konnten, war es möglich, Larven über den kritischen Punkt (völlige Resorption des Dotters) hinaus zu halten; und zwar hielten sich die Larven in denjenigen Zuchtbehältern, die nur während einiger Stunden am Tage direktes Sonnenlicht erhielten (Versuch II), am besten.

4. Aus Versuch I ließen sich einige Larven bis zu 23 Tage und aus Versuch II bis zu 40 Tage nach der Aufzehrung des Dottermaterials halten.

5. Nahrungsaufnahme konnte nur bei Larven aus den Versuchen I und II festgestellt werden, und zwar frühestens nach völliger Resorption des Dotters. Als Nahrung wurden im Darm nachgewiesen: 1. Nauplien von Copepoden, 2. Schwärmer von Grünalgen und 3. eine undefinierbare grüne Masse ("green food remains", LeBour).

6. Für das Auftreten von Gasblasen im Darm, die in der Hauptsache den Tod der meisten Larven bedingten, konnte keine Erklärung gefunden werden. Auf die Assimilationstätigkeit aufgenommener, nicht abgestorbener Phytoplanktonten können die Gasblasen nicht zurückgeführt werden; andererseits konnte niemals beobachtet werden, daß die Larven nach im Wasser verteilten Luftbläschen schnappen und diese verschlucken.

7. Die Zahl der Chordazellen schwankt zwischen 92 und 124, die der Myomeren zwischen 55 und 63 (Durchschnitt von 35 Zählungen 111.3 bezw. 58.6).

8. Eine Abhängigkeit der Zahl der Chordazellen und die der Myomeren voneinander, so wie von der Temperatur während der ersten Entwicklung konnte nicht festgestellt werden. Eine Abhängigkeit der Wirbelzahl von derjenigen der Myomeren konnte mit Sicherheit nicht nachgewiesen werden. 


\section{Sehriftennachweis.}

1. Hagmeier, A.: Züchtung wirbelloser Meerestiere. In: Abderhaldens Handbuch der biologischen Arbeitsmethoden. Bd. IX, 5. 1930.

2. HeINCKE, F.: Die Varietäten des Herings. In: Jahresberichte der Commission zur wissensehaftlichen Untersuchung der Deutschen Meere in Kiel. 1878.

3. Hertuing, H.: Züchtung von Meeresfischen. In: Abderhaldens Handbuch der biologischen Arbeitsmethoden. Bd. IX, 6. 1935 .

4. JESPER SEn, P.: Investigations on the food of the herring. In: Medd. f. Com. f. Hav., Serie: Plankton. Bd. II., 1928.

5. KupFFER, C.: Ueber Laichen und Entwicklung des Herings in der westlichen Ostsee. In: Jahresberichte der Commision zur wissensehaftlichen Untersuchung der Deutschen Meere in Kiel. 1878.

6. - : Die Entwicklung des Herings im Ei. Ebeñda.

7. Meyer, H. A.: Beobachtungen über das Wachstum des Herings. In: Jahresberichte der Commission zur wissenschaftlichen Untersuchung der Deutschen Meere in Kiel. 1878.

8. Lebour, M. V.: The food of post-Iarval fish. In: Journ. Mar. Biol. Ass., XI, 41918.

9. - : The food of $\cdot$ young fish. Ebenda, XII, 2, 1920.

10. - : The food of young Clupeoids. Ebenda, XII, 3, 1921.

11. - : The food of young herring. Ebenda, XIII, 2, 1924.

12. RunNstrem, S.: The pelagic distribution of the herring larvae in the Norwegian waters. In: Rapp. Proc. Verb. LXXXVII, 5, 1934

13. SchNAKENBECK, W.: Rassenuntersuchungen am Hering. In: Berichte der Deutschen wissenschaftlichen Kommission für Meeresforschung, N. F. 3, 1927.

14. - : Zum Rassenproblem bei den Fischen. In: Zeitschrift für Morphologie und Oekologie der Tiere. Bd. 21, 1921. 